\title{
A Review on the Efficacy of Immediacy Factors towards Achieving Communication Satisfaction via Email among Academic Staff
}

\author{
Chinedu Eugenia Anumudu \\ Mal-Imran Yasin (PhD) \\ Akmar, Ahmad Ghazali (PhD) \\ Muhammad Pauzi Latif (PhD)
}

Department of Communication, University Putra Malaysia

Doi: $10.2478 / \mathrm{mjss}-2018-0065$

\begin{abstract}
The review paper intensively explored immediacy factors influencing communication satisfaction through asynchronous computer mediated communication mediums. Immediacy is one of the constructs of social presence theory deemed capable of compelling communication satisfaction on asynchronous computer mediated communications. It has been established in numerous related studies conducted on asynchronous virtual mediated channels. However, little or no consideration seemed to have been given to exploring these immediacy factors towards ascertaining communication satisfaction via email, as one of the asynchronous mediated communications. The need to study this via email is essential, especially towards making it more efficient since it still remained the third means of communication predominantly used for dispatching corporate issues. In this review, prompt feedback, approachability and similarity personality were the dimensions of immediacy explored in relations to the effectiveness towards attaining communication satisfaction on asynchronous virtual communications. The findings showed that these dimensions examined were capable of inspiring immediacy towards achieving communication on asynchronous computer mediated channels. Therefore, it is expected that these may equally motivate communication satisfaction via email as one of asynchronous virtual mediums. It also showed that most of the studies so far conducted were dominated by qualitative and mixed-method approaches. Few quantitative studies encountered in the review appeared lacking the capacity of generalizing the outcomes due to respondents' poor turnout rate and inadequate sample sizes issues. Thus, empirical quantitative studies are still needed via email mode of interaction in order to resolve these issues and for revalidating the outcomes of the review.
\end{abstract}

Keywords: Immediacy factors efficacy; Email users; Communication satisfaction; Academic staff; asynchronous computer mediated communications

\section{Introduction}

Interpersonal communication is crucial in any corporate firms for organizing and accomplishing its tasks, which may take place either via computer mediated or non-mediated mediums. However, attention was given to asynchronous computer mediated forms of communications for the review. Generally, computer mediated communications are categorized into synchronous and asynchronous types. Synchronous computer mediated communications refer to all forms of computer mediated communications that require real-time dialogues with communicating entities 
through various communication technologies such as chat systems and telephone conversations etc., while asynchronous computer mediated communications are the ones like email, bulletin board and cell phone text messages where real-time conversations hardly exist among interacting individuals (Kawase, 2015). Consequently, this study precisely focused on email as one of the asynchronous virtual communications in order to ascertain whether immediacy dimensions may inspire communication satisfaction via it.

Email, is one of asynchronous computer mediated communications globally used for accomplishing various tasks in different corporate organizations and equally distinctively remarked as one of the main modes of communications (Swacha, 2015). Despite being referred as one of the oldest means of communications, its relevance at present time cannot be underestimated due to various duties it is still used to achieve, especially in corporate firms, thus, Backholm and Vuornos (2014) proposed that email is a mode of virtual communication mostly used for conveying messages in various organizations. Furthermore, Grevet Choi, Kumar and Gilbert (2014) pointed that email is still used for attaining some personal needs such as using it for conversing with friends, relatives; and family members; additionally, it was stated as a channel use for dispatching bill related issues as well as accomplishing group events.

Among academic staff, which is the focussed subject of the review, email is one of the internet services mostly valued and used for discharging official duties because of the lesser time consumption and inexpensive in usage as well as serving as source of information (Bankole, 2013). It was also listed among information and communication technologies academic staff found easy and often integrated in teaching profession (Tzitzolaki, Tsiligiri and Kostouda, 2014). Serrat (2017) equally observed that knowledge workers these days mostly discharge their duties via virtual offices, thus, electronic mail is used by them to strengthen and enhance work, thereby reducing phone calls by adopting electronic mail as preferred mode of interaction. Importance of email and its usages are not only limited within academic staff, it can equally be used for connecting with the majority of students who are undertaking online courses, hence, has been attested by students to be the best method of communicating with lecturers due to its convenience (Chang, Hurst and McLean, 2015). Similarly, Cruise (2016) pointed that email is one of the virtual communication media used for expediting communications between academic staff and students; therefore, it was stated to be frequently used for dialoguing between teachers and the students.

However, email has been also globally criticized as one of impersonal and lean channels of asynchronous virtual communications due to its ability to impede interpersonal relationship development while interacting via it (Wu, Gao and Zhang, 2014). Thus, communication dissatisfaction may be witnessed while communicating through it.

In reference to the above issues, some factors have been established by scholars as predictors of communication satisfaction, specifically on other asynchronous computer mediated communications but not yet through email. Consequently, immediacy concept propounded by Mehrabian (1971), which emanated from social presence theory (SPT), has been identified as an element that may effect communication satisfaction via asynchronous computer mediated communication (Kreijns, Van Acker, Vermeulen and Van Buuren, 2014). Immediacy concept on asynchronous virtual communication refers to all the communicative, verbal and non-verbal behaviours that can assist in reducing the psychological and physical distance among communicating entities as substitutes to shortage of verbal, non-verbal contacts and body language clues experienced while interacting via computer mediated communications settings (Al Ghamdi, 2017). This concept will be considered from three dimensions in this review, which are: prompt feedback, approachability and similarity personality based on the influences on communication satisfaction via asynchronous computer mediated communications according to (Asiri 2013; Lee and Lee, 2014). Therefore, this review paper covered the following immediacy dimensions questions: how does prompt feedback influence immediacy towards communication satisfaction on asynchronous computer mediated communications? What are the effects of approachability on immediacy towards communication satisfaction on asynchronous computer mediated communications? And what is the influence of similarity personality on immediacy towards communication satisfaction on asynchronous computer mediated communications? It is assumed that these scopes of immediacy in considerations to communication satisfaction on asynchronous 
virtual communications may equally influence communication satisfaction via email as one of the most valuable asynchronous computer mediated communications.

\section{Methodology}

The review paper mainly concentrated on secondary data collected from various sources, ranging from books, journal articles and conference papers proceedings. The methodology was employed because the topic area explored in the review was a diversified one.

\section{Literature review}

\subsection{How does prompt feedback influence immediacy towards communication satisfaction on asynchronous computer mediated communications?}

Prompt feedback is one of the dimensions of immediacy concept that may influence communication satisfaction on asynchronous computer mediated communication channels. Consequently, a study explored towards ascertaining the effects of changing the structures of interaction board systems in order to improve students' engagements and participations, demonstrated that commitment levels, kind attitude and unambiguous directions in written contents are the factors that can instigate prompt feedback towards achieving communication satisfaction, especially on asynchronous mediated communications (Wegmann and McCauley, 2014). Though, this study was a qualitative study carried out with 32 participants from two separate universities involving student teachers, who were undergoing online masters' programs; the sampling method and the numbers of respondents precisely selected from each of the universities seemed omitted. This could have helped in justifying the sampling method as qualitative study. Additionally, it equally appeared lacking the capacity of generalizing the findings as factors that may bring about prompt feedback for immediacy effect towards achieving communication satisfaction via asynchronous virtual communications.

Similarly, study conducted by Plante and Asselin (2014) in order to identify best implementations and proof- based policies towards making online education setting, that will incorporate caring attitude and enhance social presence; showed that quick feedback, integrating timely reply projected towards clarifying issues and responsiveness in online communication were likely to improve immediacy effect for communication satisfaction in virtual environment. Nonetheless, this study was limited to a descriptive one. Thus, an empirical study would have helped in establishing connection between the factors described in this study as predictors of prompt feedback for immediacy effect towards achieving communication satisfaction. Non-verbal behaviours such as giving rapid or moderate feedback, expediting of jobs and procedures of discussions, have been also presented to have abilities of enhancing social presence and stimulating immediacy outcome towards achieving communication satisfaction on virtual world (Zydney, 2014). Nevertheless, these were observed via a conceptual paper involving students and their instructors while undertaking online courses, thus, may be said to be lacking empirical evidence in order to qualify it as an empirical study.

Whereas Walkem (2014) differed by presenting that the ability to attain immediacy in elearning for the purpose of effecting prompt feedback towards satisfaction, involved the following themes: recognizing and discharging professional duties, conveying precise and well-timed messages; and employing a rich media. Notwithstanding that qualitative data are deemed richer; the study was equally limited to fewer nursing students and lecturer in online learning. Therefore, it may be said that it lacked the capacity of generalizing the outcome due to limited numbers of respondents. Similarly, Conrad (2016) argued that feedback on asynchronous virtual medium can only be useful in motivating immediacy outcome towards reaching communication satisfaction if the contents were personalized, unambiguous and well-timed. Despite these findings, the study was equally had smaller numbers of students and instructors on asynchronous learning environs as its respondents. Furthermore, mixed- methods employed in this study threatened the validity of the data because; the real feedback impact on students' activities was neglected in the assessment items. Hence, this study may have generated a good validity result if some items were specifically 
set out for measuring prompt feedback itself.

However, expediting feedbacks has been proposed by Guo, Chen, Lei and Wen (2014) as positively influencing the rate of cognitive commitment on virtual environment for the purpose enhancing immediacy result towards instigating communication satisfaction. Even though that this finding was observed in mixed method, participants were also limited to only two classes of distant learners who were undertaking online training career. It may have given more reliable outcome if more than two online trainers from other institutions were equally involved. Contrarily, it has been contested by Cunningham (2015) that using other means such as automated avatars to substitute social presence on virtual communication setting does not bring about closeness or satisfaction, rather, faster responses from the interacting persons, may assist in achieving immediacy effect towards obtaining satisfaction and sense of belonging on asynchronous virtual world. This was observed in qualitative study's content analysis triangulated by both experiment and opened-ended questions' methods for more reliable result. Nevertheless, the outcome was made from a study conducted among only 40 participants. Therefore, there may be tendency of producing a countered outcome if a research method that will incorporate larger sample size was used. Research also illustrated that communication satisfaction via online asynchronous virtual dialogue entailed having suitable and frequent feedback; perfect directions; meaningful discussions; and cannot just be achieved through only rapid feedback (Hung and Chou, 2014). However, the data of this study appeared lacking criterion validity due to its exploratory nature that made the researcher to finalize the result based on only two sample sets. Thus, it was assumed that if more than two sample set were involved in the study, it would have produced better criterion in terms of its validity. Furthermore, Shen and Chen (2014) added that providing both regular and helpful feedback at a suitable time, assisted in enhancing immediacy towards communication satisfaction, especially on asynchronous computer mediated communication where the physical presence of the communicating individuals was lacked. Due to the fact that this qualitative study was carried out via only interviews among just seven numbers of online dissertation doctorate students, it may have threatened the validity and reliability outcome of this study as qualitative study. Therefore, more than one data collection approaches would have been triangulated for the purpose of getting more valid and reliable outcomes.

Karahoca, Karahoca and Kurnaz, (2016) also contended the notion that virtual communicators specifically derive communication satisfaction when there were prompt feedbacks but added that motivation towards replying on time can be improved once the contents of the messages were understandable and sent from familiar persons. Even though that the findings were realized through a quantitative method, it was limited to only Bahcesehir Turkish undergraduates who were undergoing online engineering ethics course and can only be generalized among these particular undergraduates. Thus, it is assumed that expanding the study to other universities' undergraduates in Turkey would have made it capable of generalizing the findings among other Turkish undergraduates.

\subsection{What are the effects of approachability on immediacy towards communication satisfaction on asynchronous computer mediated communications?}

Approachability as one of the dimensions of immediacy equally plays much role towards immediacy outcomes for communication satisfaction on asynchronous virtual communications. Thus, Richardson, Besser, Koehler, Lim and Strait (2016) demonstrated that communication approachability patterns on asynchronous online discussions such as moderating the tone of the discussion, coupled with exhibiting anticipated behaviours and various personal manners may influence participants' engagements and satisfaction towards immediacy result. However, these qualitative findings were established through interviews limited to 13 numbers of online instructors who handled online masters' programs, therefore, it may have produced a better valid data if two or more data collection methods were triangulated. Additionally, it seemed lacking the capacity of generalizing the outcomes of this study due to the research method and sample size involved.

Approachability on asynchronous computer mediated medium has been also referred to as verbal behaviours that indicated to others that one is well disposed and friendly to communicate 
with (Pang, Shin, Lew and Walther, 2016). This incorporated manners that may strengthen bond between virtual communicating entities. These behaviours conspicuously signified accessibility, which may bring about immediacy effect towards achieving satisfaction on asynchronous computer mediated mediums. Nonetheless, since the study was a qualitative one, it has only few organizations and stakeholders that participated in it. Therefore, the outcome may have been influenced by organizational background, thus, decentralizing studies that cannot be influenced by certain organizational orientations are suggested. Furthermore, approachability as scope of immediacy towards achieving satisfaction on virtual mediated discussions was claimed by Hughes (2014) to be influenced by the manners in which the content of the discussion was presented to fellow communicating partners. In other words, this implied that when better approachability is shown to other users of asynchronous virtual communication media, the more motivated they will be, especially towards remaining firm in interacting via same media. Nonetheless, this was established from a quantitative survey study that incorporated only undergraduates who engaged in 6 various online classes in Western Kentucky University. Therefore, the outcome may be biased since it was conducted in just one particular university. Moreover, it seemed lacking the capacity of generalizing the outcome among other students of this particular university since the majority that participated in the study comprised first year students. Thus, there is need to extend similar study to students of other levels in this university and also by using a sampling technique that will not give a biased selection. Additionally, the study can equally be extended to students from other universities to avoid questioning the capability of generalizing the findings. Similarly, Roberts and Friedman (2013) viewed approachability part of immediacy as an act of displaying friendly attitudes via the manner by which discussion was presented to other interacting individuals for achieving effective participatory dialogue. However, this dimension of immediacy was conceptualized through face face class interaction between teachers and students. Moreover, it looked limited across disciplinary sampling carried out via objective that only allowed observation process. Therefore, there are necessities to confirm the findings of this study via asynchronous computer mediated communications since it was limited to face-face communication and also a quantitative approach may be equally employed for the purpose of integrating larger sample size and generalizing the outcomes.

Approachability towards immediacy effect for communication satisfaction in online discussion has been also viewed from other contrarily perspectives by (Brooks and Young, 2016). These involved displaying unaccommodating behaviours such as: uninspiring, intolerant, hasty, and unfriendly attitudes among virtual communicating partners. Nevertheless, these were hypothesized via quantitative online surveys conducted among only undergraduates, who were engaged in outside classrooms' learning. Therefore, it may be marred by wide sweep of data collection. This may be as a result of employing data from students taught by various instructors and might have been influenced by different teachers' approaches. Therefore, it may be assumed that wide sweep of data collection witnessed in this study would have been avoided if all the respondents evaluated went through online learning under the same instructor. Research also illustrated that the act of presenting selves on virtual communication as humane through the content of the dialogue on computer mediated communication assisted in minimizing the psychological and physical distance for immediacy effects towards achieving communication satisfaction (Barkey, 2014). Irrespective of these findings, this study appeared to limit its respondents to fewer numbers, even though it employed mixed method research for triangulating and validating its data. Moreover, it equally looked limited to instructors and students who were undergoing a distant learning course in a particular institution. Hence, extending the study to students from other universities would have offered more sample size and capacity of generalizing the study's outcomes within the study areas. Approachability scope of immediacy on asynchronous computer mediated communications was also said to be determined by some factors such as communicating parties' discernments on individuals' accessibility, trustworthiness; and professionalisms as well as communal connections among the interacting persons (Sarapin and Morris, 2015). However, this study was specifically limited to instructors and students via faceboook medium of interaction and equally seemed to be marred by low response turnout rate, in spite being a quantitative study. The low turnout rate might have been caused by ambiguities on the study's instrument or lack of awareness on why the 
respondents should have participated in it. Therefore, future similar study will ensure that the items in the research questionnaire are well clarified for a layman's understanding. Additionally, adequate enlightenment on why the respondents should consider it useful for partaking in the study should equally be given much attention. Schroeder, Baker, Terras, Mahar and Chiasson (2016) also demonstrated that approachability for immediacy effect towards communication satisfaction on asynchronous mediated communication can be enriched by increasing social presence via the degree of connectivity among the interacting entities. Though this was realized via a quantitative study conducted among teachers and distant masters' degree learners, the numbers of the respondents who willingly participated in the study seemed inadequate to generalize the outcome. Hence, subsequent studies on similar area should concentrate on enlarging the sample size and also in creating much awareness on why the respondents will see it as a valuable exercise for participation.

\subsection{What are the influences of similarity personality on immediacy towards communication satisfaction on asynchronous computer mediated communications?}

Similarity in personality was also conceptualized as one of immediacy dimensions that may bring about immediacy towards reaching communication satisfaction on asynchronous computer mediated communications, hence, Kirwan and Roumell (2015) presented that personal behaviours, which corresponded with virtual communicating partners' characters may instigate immediacy effect towards achieving communication satisfaction on virtual settings. However, this study was a mere conceptual paper; therefore, it may be lacking empirical evidence. Hence, empirical studies are still needed in order to scientifically justify the findings already identified in this study. Showing mutual human personalities such as integrating symbiotic emotional and instilling attitudes while communicating via virtual media was also stated by McCool (2016) as having the ability of enhancing social presence for immediacy effect towards attaining gratification. Though these were observed from grounded theory qualitative approach, the study specifically appeared limited to fewer instructors and students who were undergoing writing learning programs via communication technology. Therefore, prospective studies should precisely concentrate on studying any other asynchronous computer mediated communications such as email for the purpose of exploring the effects of similarity personality scope of immediacy towards ascertaining communication satisfaction. Furthermore, another limitation was omitting to collect data from freshmen, who were also involved in online composition course in the same institution; and this may have made the data collected in this study a biased one. Hence, this can be readdressed by employing a particular sampling method when selecting the sample sizes in subsequent studies in order to justify the sample sizes.

Research also established that adapting to similar personalities brings about immediacy effect towards obtaining communication satisfaction in either virtual or non-virtual environments $(\mathrm{Bi}$, Fussell and Birnholtz, 2014). However, it also presented that cultural backgrounds may serve as a mediator predictor in such study. Nevertheless, this qualitative study was experimented among 50 Chinese and United State locals working in United Sate; therefore, it might have been biased by United State cultural background since it was conducted there. Consequently, there is need to reconfirm the findings of this study with different respondents in a neutral setting to avoid being influenced by cultural settings. Observing politeness and conscientiousness personalities with virtual communicating individuals, especially when interacting with older team workers was also proposed by Valdez, Schaar and Ziefle (2013) to have the capability of influencing immediacy result towards attaining satisfaction on social network. Nevertheless, this quantitative study seemed to be conducted with inadequate numbers of younger and inexperienced respondents who were still lacking technicalities from educational backgrounds. Furthermore, the findings may be biased by German cultural views since it was conducted there. Therefore, it is assumed that conducting similar study with larger technically experienced respondents and on neutral settings may help in resolving the issue of biased cultural result and may equally make the study capable of generalizing its findings. Research also demonstrated that displaying similar personalities such as self-esteem attitude and responsiveness in dialoguing on social networking can influence immediacy result 
towards improving social presence for communication satisfaction (Alshuaib, 2014). Despite that this study employed mixed- method and quasi-experiment for enriching its data, it was conducted only among local students who engaged in online class discussion at the university of Exeter, Kuwaiti higher education context, therefore, the use of only locals as the same study's respondents may be influenced by specific cultural native context and inadequate numbers of the participants may also impede generalizing the outcome of this study within the study's setting. Hence, subsequent studies need to be conducted at unbiased settings with combinations of other ethnicities in order to resolve cultural influences observed in this study. Additionally, there is need to employ a quantitative approach in future studies for the purpose of enlarging the respondents in order to generalize the outcomes within the study areas. Similarly, Rutjes (2013) demonstrated that related personalities possessed by individuals who were interacting via particular mode of communication technology may equally influence satisfaction. This implied that having matching characters with fellow communicating partners on virtual mediated communication will probably help in minimizing the psychological and physical distance between interactants for immediacy outcome towards achieving communication satisfaction. However, this quantitative research was only an outcome of experimental study between a single robot and human beings; therefore, there is need to extend similar study among fellow human beings via asynchronous virtual communications since this review precisely concentrated on human beings. Research equally pointed that collaborations and contentment can instigate immediacy effect towards attaining communication satisfaction if both virtual communicating individuals possess similar personalities such as mutual relationships, trust and having abilities of sharing common understanding irrespective of one's cultural background (Krumm and Schulze, 2017). Nonetheless, these were identified in a mere book section analysis, therefore, may be said to lack empirical fact. Thus, the findings need to be justified through employing empirical studies.

\section{Conclusion and Perspective}

This review has shown that the three immediacy dimensions, incorporating prompt feedback, approachability and similarity personality were capable of influencing immediacy results towards achieving communication satisfaction on asynchronous computer mediated communications.

However, the reviews also showed that most studies already conducted in this field of study appeared limited to some asynchronous computer mediated communications mediums. Based on these findings, it seemed little or no study have been specifically conducted through email in order to ascertain if these immediacy dimensions may equally enhance communication satisfaction via it since email, has been identified as one of the asynchronous computer mediated communications primarily used for discharging official issues even among academic staff. Hence, there is need to empirically conduct a study via email in order to ascertain if these immediacy dimensions may equally make it more efficient towards achieving communication satisfaction through it. Thus, it is expected that those immediacy factors may also influence communication satisfaction while interacting via it.

Furthermore, this review equally showed that most studies already conducted on this field of study seemed dominated by qualitative and mixed- method research approaches. Little studies, which were conducted through quantitative methods so far, appeared to have low turnout rates from the respondents; while some had inadequate sample sizes issues and use of only respondents from particular institutions/firms. Therefore, there is still lack of quantitative studies incorporating larger sample sizes from diverse places and respondents for the purpose of empirically validating most findings in this review and also for making the studies capable of generalizing the outcomes. Consequently, it is suggested that further empirical studies should be conducted through quantitative approach towards ascertaining if these immediacy dimensions may also enhance communication satisfaction specifically via email among academic staff. 


\section{References}

Al Ghamdi, A. (2017). Influence of Lecturer Immediacy on Students' Learning Outcomes:

Evidence from a Distance Education Program at a University in Saudi Arabia. International Journal of Information and Education Technology, 7(1), 35.

Alshuaib, A. (2014). Promoting Social Presence in a Social Networking Environment in a Kuwaiti Higher Education Context.

Asiri, A. A. (2013). The Impact of Instructor Immediacy on College Student Communication and Learning Outcomes in Saudi Arabia(Doctoral dissertation, Victoria University).

Backholm, A., \& Vuornos, L. (2014). U.S. Patent No. 8,738,050. Washington, DC: U.S. Patent and Trademark Office.

Bi, N., Fussell, S. R., \& Birnholtz, J. (2014, August). To be like you to be liked by you: cultural effects on adjusting awareness information gathering behavior. In Proceedings of the 5th ACM international conference on Collaboration across boundaries: culture, distance \& technology (pp. 31-40). ACM.

Bankole, O. M. (2013). The use of internet services and resources by scientists at Olabisi Onabanjo University, Ago Iwoye, Nigeria. Program, 47(1), 15-33.

Barkey, D. W. (2014). Student perceptions of instructor credibility based on instructor communication in an exclusively internet-based learning environment (Doctoral dissertation, Argosy University/Phoenix).

Brooks, C. F., \& Young, S. L. (2016). Exploring Communication and Course Format: Conversation Frequency and Duration, Student Motives, and Perceived Teacher Approachability for Out-of-Class Contact. The International Review of Research in Open and Distributed Learning, 17(5).

Conrad, S. (2016). Student and Instructor Perceptions of Feedback in Asynchronous Online Learning: A MixedMethods Study (Doctoral dissertation, George Mason University).

Chang, C. W., Hurst, B., \& McLean, A. (2015). You've got mail: Student preferences of instructor communication in online courses in an age of advancing technologies. Journal of Educational Technology Development and Exchange (JETDE), 8(1), 3.

Cunningham, J. M. (2015). Mechanizing People and Pedagogy: Establishing Social Presence in the Online Classroom. Online Learning, 19(3), 34-47.

Cruise, R. (2016). School of Computing.

Grevet, C., Choi, D., Kumar, D., \& Gilbert, E. (2014, April). Overload is overloaded: email in the age of Gmail. In Proceedings of the sigchi conference on human factors in computing systems(pp. 793-802). ACM.

Guo, W., Chen, Y., Lei, J., \& Wen, Y. (2014). The effects of facilitating feedback on online learners' cognitive engagement: Evidence from the asynchronous online discussion. Education Sciences, 4(2), 193-208.

Hung, M. L., \& Chou, C. (2014). The development, validity, and reliability of communication satisfaction in an online asynchronous discussion scale. The Asia-Pacific Education Researcher, 23(2), 165-177.

Hughes, G. K. (2014). The effect of mediated immediacy upon state motivation and cognitive learning in an online lesson. University of Kentucky.

Karahoca, D., Karahoca, A., \& Kurnaz, A. (2016). Analyzing Communication Dimensions in a Ubiquitous Learning Environment. J. UCS, 22(1), 124-145.

Kawase, A. (2015). Second language acquisition and synchronous computer mediated communication. Teachers College, Columbia University Working Papers in TESOL \& Applied Linguistics, 6(2).

Kirwan, J. R., \& Roumell, E. A. (2015). Building a Conceptual Framework for Online Educator Dispositions. Journal of Educators Online, 12(1), 30-61.

Kreijns, K., Van Acker, F., Vermeulen, M., \& Van Buuren, H. (2014). Community of inquiry: Social presence revisited. E-learning and Digital Media, 11(1), 5-18.

Krumm, S., \& Schulze, J. (2017). Work and Virtual Collaboration. The Wiley Blackwell Handbook of the Psychology of the Internet at Work, 7696, 61.

Lee, H. S., \& Lee, H. J. (2014). Factors Influencing Online Trust. Academy of Marketing Studies Journal, 18(1), 41.

McCool, L. B. (2016). Humanizing advanced communication online writing instruction: Developing social presence to communicate, collaborate, and connect (Doctoral dissertation, lowa State University).

Mehrabian, A. (1971). Silent messages (Vol. 8). Belmont, CA: Wadsworth.

Pang, A., Shin, W., Lew, Z., \& Walther, J. B. (2016). Building relationships through dialogic communication: organizations, stakeholders, and computer-mediated communication. Journal of Marketing Communications, 1-15.

Plante and Asselin (2014). Best practices for creating social presence and caring behaviors online. Nursing Education Perspectives, 35(4), 219-223.

Richardson, J. C., Besser, E., Koehler, A., Lim, J., \& Strait, M. (2016). Instructors' perceptions of instructor presence in online learning environments. The International Review of Research in Open and Distributed Learning, 17(4). 
Roberts, A., \& Friedman, D. (2013). The Impact of Teacher Immediacy on Student Participation: An Objective Cross-Disciplinary Examination. International Journal of Teaching and Learning in Higher Education, 25(1), 38-46.

Rutjes, J. A. (2013). Expectations and Human-Robot Interaction. The influence of robot expectations on personality attribution, impressions and anthropomorphism (Master's thesis, University of Twente).

Sarapin, S. H., \& Morris, P. L. (2015). Faculty and Facebook friending: instructor-student online social communication from the professor's perspective. The Internet and Higher Education, 27, 14-23.

Serrat, O. (2017). Managing by walking around. In Knowledge Solutions (pp. 321-324). Springer Singapore.

Shen, L., \& Chen, I. L. (2014). Social presence in online dissertation classes. Educational, psychological, and behavioral considerations in niche online communities, 175-191.

Schroeder, S., Baker, M., Terras, K., Mahar, P., \& Chiasson, K. (2016). Desired and Experienced Levels of Connectivity to an Asynchronous, Online, Distance Degree Program. Online Learning, 20(3).

Swacha, J. (2015). Management by Email Reinterpreted with a Process-Based Approach. In Managing Intellectual Capital and Innovation for Sustainable and Inclusive Society: Managing Intellectual Capital and Innovation; Proceedings of the MakeLearn and TIIM Joint International Conference 2015 (pp. $1343-$ 1350). ToKnowPress.

Tzitzolaki, T., Tsiligiri, M., \& Kostouda, M. (2014). The use of information and communications technology from the educational staff of the Nursing and Physiotherapy Departments of the Alexander Technological Educational Institution of Thessaloniki, Greece. Int J Caring Sci, 7(1), 203-210.

Walkem, K. (2014). Instructional immediacy in elearning. Collegian, 21(3), 179-184.

Wegmann, S. J., \& McCauley, J. K. (2014). Investigating Asynchronous Online Communication: A Connected Stance Revealed. Journal of Asynchronous Learning Network, 18(1), n1.

Wu, H., Gao, J., \& Zhang, W. (2014). Chinese EFL teachers' social interaction, socio-cognitive presence in synchronous computer-mediated communication.

Valdez, A. C., Schaar, A. K., \& Ziefle, M. (2013). Personality influences on etiquette requirements for social media in the work context. In Human Factors in Computing and Informatics(pp. 427-446). Springer, Berlin, Heidelberg.

Zydney, J. (2014). Strategies for creating a community of inquiry through online asynchronous discussions. Journal of online learning and teaching, 10(1), 153. 